\title{
Do sleep disturbances in depressed adolescents improve following psychological treatment for depression?
}

Article

Accepted Version

Creative Commons: Attribution-Noncommercial-No Derivative Works 4.0

Reynolds, S., Orchard, F., Midgley, N., Kelvin, R., Goodyer, I. and the IMPACT consortium, (2020) Do sleep disturbances in depressed adolescents improve following psychological treatment for depression? Journal of Affective Disorders, 262. pp. 205-210. ISSN 0165-0327 doi:

https://doi.org/10.1016/j.jad.2019.10.029 Available at https://centaur.reading.ac.uk/87014/

It is advisable to refer to the publisher's version if you intend to cite from the work. See Guidance on citing.

To link to this article DOI: http://dx.doi.org/10.1016/j.jad.2019.10.029

Publisher: Elsevier

All outputs in CentAUR are protected by Intellectual Property Rights law, including copyright law. Copyright and IPR is retained by the creators or other copyright holders. Terms and conditions for use of this material are defined in the End User Agreement. 


\section{CentAUR}

Central Archive at the University of Reading

Reading's research outputs online 
Do Sleep Disturbances in Depressed Adolescents Improve Following Psychological Treatment for Depression?

Shirley Reynolds ${ }^{1}$, Faith Orchard $^{1}$, Nick Midgley ${ }^{2,3}$, Raphael Kelvin ${ }^{4}$, the IMPACT consortium $\dagger$, and Ian Goodyer ${ }^{4}$

${ }^{1}$ School of Psychology and Clinical Language Sciences, University of Reading

${ }^{2}$ Child Attachment and Psychological Therapies Research Unit (ChAPTRe), Anna Freud National Centre for Children and Families, London, UK

${ }^{3}$ Research Department of Clinical, Educational and Health Psychology, University College London, London, UK

${ }^{4}$ Department of Psychiatry, University of Cambridge, Cambridge, UK

Corresponding Author: Shirley Reynolds (ORCID: 0000-0001-9975-2023)

School of Psychology and Clinical Language Sciences

University of Reading, Reading, RG6 6AL, UK

Telephone +44 (0)118 3788525

Email: s.a.reynolds@ reading.ac.uk

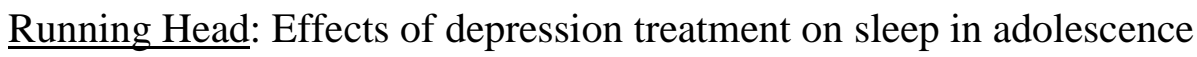

Conflicts of Interest: The authors declare no conflicts of interest.

$\dagger$ Members of the IMPACT Consortium: Ian M Goodyer, Shirley Reynolds, Barbara Barrett, Sarah Byford, Bernadka Dubicka, Jonathan Hill, Fiona Holland, Raphael Kelvin, Nick 
Midgley, Chris Roberts, Rob Senior, Mary Target, Barry Widmer, Paul Wilkinson, and Peter Fonagy. 


\begin{abstract}
Background. Persistent sleep disturbances are one of the most common symptoms of Major Depressive Disorder (MDD) in adolescence. These are not typically targeted in psychological treatments and it is not known if psychological treatment for depression improves sleep.

Methods. Secondary analyses were conducted using data from a large, multi-centre, randomised controlled trial (Goodyer et al., 2017b). Young people aged 12-18 years $(\mathrm{N}=$ $465 ; 75 \%$ female) met diagnostic criteria for Major Depressive Disorder, based on the Kiddie-Schedule for Affective Disorders and Schizophrenia (K-SADS). They were randomised to one of three psychological treatments. Sleep difficulties were assessed at baseline, post-treatment (36 weeks) and one year follow up (86 weeks) with the K-SADS, and the Mood and Feelings Questionnaire (MFQ).
\end{abstract}

Results. At baseline, $92 \%$ of young people exhibited clinically significant sleep difficulties. Exploratory analyses suggest that sleep difficulties significantly decreased from baseline to end of treatment on self-report and interview-based measures, and this decrease was maintained at follow up. Reduction in sleep difficulties did not differ between the psychological treatments. Approximately, half of young people reported residual sleep difficulties at the end of treatment and at follow-up.

Limitations. This paper reports secondary data analyses and findings are exploratory. Conclusions. Tentative results suggest that psychological treatments for depression reduced sleep problems for some participants. However, young people with treatment-resistant sleep problems may benefit from adjunctive sleep interventions. Future work with a range of sleep measures is needed to determine those who have residual sleep problems at the end of treatment and post-treatment follow-up.

Keywords: Depression; Sleep; Psychotherapy; Adolescence 


\section{Introduction}

Sleep problems are common in teenagers (Gradisar, Gardner, \& Dohnt, 2011;

Ohayon, Roberts, Zulley, Smirne, \& Priest, 2000). These are believed to be due to a range of social factors and to changes in the circadian rhythm during puberty (Owens, 2014).

Common sleep difficulties amongst the general adolescent population typically include insufficient sleep (Eaton et al., 2010) and weekday-to-weekend sleep variability (Wittmann, Dinich, Merrow, \& Roenneberg, 2006). Sleep problems can become chronic and persistent during adolescence (Sivertsen, Harvey, Pallesen, \& Hysing, 2017), and can be linked to poor emotional and physical health and impaired functioning at home and school (Shochat, CohenZion, \& Tzischinsky, 2014), as well as being distressing and disruptive for the whole family (Wake et al., 2006).

These persistent sleep problems are often experienced by individuals with worry and low mood (Gregory \& O'Connor, 2002). As part of a diagnosis of Major Depressive Disorder (MDD), adolescents are required to present with at least one 'core' symptom, and a selection of further additional symptoms. One of the possible additional symptoms is 'sleep disturbance', and this has been found to be one of the most common symptoms of depression in adolescents (Orchard, Pass, Marshall, \& Reynolds, 2017). The 'sleep disturbance' symptom is broken down into depression-specific sleep difficulties, including insomnia (not being able to get to sleep), hypersomnia (sleeping too much), circadian reversal (being active at night and sleeping during the day) and non-restorative sleep (not feeling rested after sleep ) (APA, 2000). This range of difficulties are not typically experienced by the general adolescent population, or by adolescents with other common mental health problems such as anxiety, where individuals primarily experience difficulties with insomnia (Alfano, Ginsburg, \& Kingery, 2007). Furthermore, depressed young people who report problems with sleep have been found to be more likely to have severe depression (Liu et al., 2007) and to have 
thoughts about death and suicide (Urrila et al., 2012) than those who do not have problems sleeping. Sleep disturbances have also been found to be a common residual symptom among depressed adolescents (Kennard et al., 2006) and adults (Carney, Segal, Edinger, \& Krystal, 2007; Nierenberg et al., 2010), and residual insomnia has been found to increase the risk of depression recurrence (Dombrovski et al., 2008).

Some authors have suggested that sleep problems are not just a symptom or a byproduct of depression, but that they may contribute to the onset or maintenance of depression (Clarke \& Harvey, 2012). Most of this work specifically examines insomnia. This is mainly because insomnia is considered the most common sleep disorder in youth (e.g. Johnson, Roth, Schultz, \& Breslau, 2006; Ohayon et al., 2000), and because insomnia treatments are well-established i.e. Cognitive-Behavioural Therapy for Insomnia (CBTi). There is also evidence that sleep difficulties in children and young people are likely to predict the risk of developing depression in later childhood or adulthood (de Bruin, Bögels, Oort, \& Meijer, 2015; Gradisar et al., 2011; Roane \& Taylor, 2008; Roberts \& Duong, 2013). There may be different causal pathways or common factors associated with the onset of both sleep problems and depression, for example physical problems linked to chronic illnesses (e.g. pain, problems breathing), or negative life events or adverse socio-economic circumstances. This possible causal relationship, and the focus on insomnia, has led to the proposal of specific guidelines for treating 'insomnia' in adolescence, i.e. drawing on techniques from CBTi (Clarke \& Harvey, 2012). It is also important to note that while sleep disturbance is a diagnostic criterion for MDD, sleep disorders (specifically insomnia disorder and delayed sleep-wake phase disorder) are conceptualised as independent diagnostic entities (Staner 2010) and are often comorbid with depression (Buysse et al., 2008).

Psychological interventions based on cognitive behavioural models of insomnia are an effective treatment for sleep problems in adults (Koffel, Koffel, \& Gehrman, 2015; Trauer, 
Qian, Doyle, Rajaratnam, \& Cunnington, 2015) and adolescents (de Bruin et al., 2015), including those with a range of mental health problems (van Straten et al., 2017; Wu, Appleman, Salazar, \& Ong, 2015). Sleep interventions can be delivered in a variety of ways, including via computer (Cheng \& Dizon, 2012), thus significantly increasing access to treatment. A recent meta-analysis of non-pharmacological sleep interventions also found that treatment for sleep problems significantly reduced symptoms of depression (Gee et al., 2018), even though the interventions did not address any other aspects of depression including core symptoms of e.g. low mood or anhedonia. The effect of sleep interventions on depression symptoms was larger in studies that recruited participants with current mental health difficulties (and hence more severe symptoms of depression). There was however limited research on the effect of treating sleep problems on depression in children and adolescents.

Just as treatment for sleep problems may improve symptoms of depression, so it is plausible that treatment for depression may improve sleep difficulties, particularly given that sleep difficulties are one of the symptoms used to diagnose Major Depressive Disorder (DSM-V; APA, 2013; ICD-10; WHO, 1992). Although sleep problems are extremely common amongst depressed young people (Orchard et al., 2017), most standardised manuals for psychological treatments for depression do not typically include sleep intervention techniques (e.g. Brent \& Poling, 1997; Martell, Addis, \& Jacobson, 2001; Ritschel, Ramirez, Jones, \& Craighead, 2011). However, the improvement of other symptoms of depression may have an indirect benefit on sleep. For example, perseverative and ruminative thinking is common in depression and is hypothesised to interfere with sleep onset (Lovato \& Gradisar, 2014). Therefore, reducing perseverative and ruminative thinking may help make sleep onset more rapid. In addition, subjective report of specific sleep problems may be inflated by negative cognitive biases or self-critical thinking, which are pervasive in depression in adults (Joorman, Yoon, \& Zetsche, 2007) and adolescents (Orchard, Pass, \& Reynolds, 2016; Platt, 
Waters, Schulte-Koerne, Engelmann, \& Salemink, 2017). Objective and subjective measurements of sleep are poorly correlated amongst depressed individuals (Bertocci et al., 2005; Dahl \& Puig-Antich, 1990) and this may be explained by potential reporting biases, such as misperception of sleep deficit, cognitive inflexibility, and selective attention and selfmonitoring (Hiller, Johnston, Dohnt, Lovato, \& Gradisar, 2015), which are likely to be amplified by low mood and worry. Successful treatment for depression reduces the associated negative cognitive biases (Tang, DeRubeis, Beberman, \& Pham, 2005) and this may lead to more favourable reports of sleep quality (Lofthouse, Gilchrist, \& Splaingard, 2009).

It was recently found that cognitive-behavioural therapy for anxiety in children and adolescents improved self-reported sleep problems (Peterman et al., 2016). However, this was only found for parent report, not child report. The authors also noted that child-reported sleep problems were low in this sample. Surprisingly, given the high prevalence of sleep problems in adolescents with depression, and the adverse impact of sleep difficulties, research has not yet examined whether treatments for adolescent depression improve sleep problems. Although there is evidence that CBT for insomnia reduces insomnia and may have an added benefit of reducing symptoms of depression, it is not known if CBT for depression improves sleep disturbances, or what effect other psychological treatments for depression have on sleep disturbances e.g. via reduction in other underlying common factors.

This paper reports on the exploratory post-hoc analyses of data from a randomised controlled trial for adolescent depression where there was high baseline prevalence of sleep symptoms (Goodyer et al., 2017b). Given the absence of evidence on this important subject, the aim of this study is twofold: first to describe the nature of sleep problems in a large sample of young people with depression, and second to examine the effect of three distinct psychological treatments for adolescent depression on sleep problems, measured by selfreport depression questionnaires and diagnostic interviews. 


\section{Methods}

\section{Participants and Recruitment}

Young people in this study were taking part in a large, multi-centre, randomised controlled trial; the HTA-funded IMPACT (Improving Mood through Psychoanalytic and Cognitive-Behavioural Therapy) Study (Goodyer et al., 2017b). IMPACT is a pragmatic superiority trial comparing the relative clinical and cost effectiveness of three psychological treatments for adolescent Major Depressive Disorder. Participants were recruited and treated at 15 National Health Service child and adolescent mental health service (CAMHS) clinics in three regions of England.

A total of 465 young people were randomised to one of three treatment approaches; Short-Term Psychoanalytic Psychotherapy (STPP); Cognitive Behavioural Therapy (CBT); and a manualised form of specialist clinical care termed Brief Psychosocial Intervention (BPI) which was chosen as the active control treatment. Lengths of treatment varied for the three approaches: 28 sessions over 30 weeks for STPP; 20 sessions over 30 weeks for CBT; and 12 sessions over 20 weeks for BPI. The three treatments were based on distinct theoretical models and were delivered in routine services by specialist clinicians according to treatment manuals (IMPACT Study CBT Sub-Group, 2010; IMPACT Study Child Psychotherapy Sub-Group, 2010; Kelvin et al., 2010). CBT and STPP manuals did not specifically consider how to address problems with sleep. The BPI manual identified sleep hygiene as a routine intervention that clinicians could offer. Treatment manuals were developed for each modality and via examination of treatment adherence using the Comparative Psychotherapy Process Scale (Hilsenroth, Blagys, Ackerman, Bonge, \& Blais, 2005), treatment fidelity was found to be good, (Midgley et al., 2018).

The mean age of participants was 15.6 years $(\mathrm{SD}=1.4)$ and $348(75 \%)$ were female. Most (85\%) described their ethnicity as White British. Participants were recruited to the study 
and treated in 15 Child and Adolescent Mental Health services in the UK. All participants met diagnostic criteria for moderate to severe Major Depressive Disorder, based on the KSADS (see below; Kaufman et al., 1997).

The study was approved by the Cambridgeshire 2 Research Ethics Committee (reference 09/H0308/137) and local NHS provider trusts. All patients and their parents gave written informed consent. See Goodyer et al. (2017a) for comprehensive information on the recruitment process and study design.

\section{Measures and Procedure}

Two measures were used to address the aims of this study: the interviewer administered Kiddie-Schedule for Affective Disorders and Schizophrenia (K-SADS-PL; Kaufman et al., 1997) and the adolescent version of the self-report Mood and Feelings Questionnaire (MFQ; Costello \& Angold, 1988). The present analyses report data from three time points from the IMPACT trial: baseline, post-treatment (36 weeks after randomisation), and at one-year follow-up (86 weeks after randomisation). Time between randomization and first session varied as treatments were delivered in routine clinical practices. Median number of weeks across location and treatment ranged from 2.9 weeks to 7.3 weeks (Goodyer et al., 2017a)

The Kiddie-Schedule for Affective Disorder and Schizophrenia Present and Lifetime (K-SADS-PL) version is a semi-structured interview measure, which was used to establish the presence of DSM-IV diagnoses. Adolescents and parents/guardians completed the measure and both interviews were used to construct a diagnosis based on positive symptom reporting from either respondent. See Goodyer et al. (2017b) for information regarding interrater reliability. The K-SADS DSM-IV Major Depressive Disorder section examines the presence or absence of all symptoms of depression in the past two weeks. Six sleep disturbance symptoms are assessed in this section: 'initial insomnia', 'middle insomnia', 
'terminal insomnia', 'hypersomnia', 'circadian-reversal' and 'non-restorative sleep'. Specific thresholds are provided by the manual to establish when participants meet criteria for each sleep symptom. For example, 'initial insomnia' = two hours or more to fall asleep, most nights. The presence of individual disturbances, as well as the total number of disturbances, is reported in the analyses. The latter is used as in indication of severity of overall sleep disturbance.

The Mood and Feelings Questionnaire (MFQ) is a 33-item self-report measure completed by the adolescent, examining depressive symptoms present over the past two weeks. The instrument is designed to cover symptom areas specified in DSM-IV for an episode of MDD, e.g. sleep disturbance. To be consistent with other measures in the IMPACT study, the MFQ was scored on a four-point scale (0-3). Ratings of '2' and ' 3 ' were then combined to be in line with typical MFQ scoring procedures. The MFQ has good testretest reliability (Pearson's $r=0.78$ ), an $\alpha$ coefficient of 0.82 and discriminant validity for detecting an episode of Major Depressive Disorder in clinical adolescent samples. Of particular interest in this paper are the two sleep items: 'I didn't sleep as well as I usually sleep' and 'I slept a lot more than usual'. These items are designed to tap into the two key DSM Major Depressive Disorder sleep symptoms; respectively insomnia and hypersomnia (APA, 2013). For the purpose of this report, these scores will be referred to as the 'insomnia' and 'hypersomnia' items.

\section{Results}

\section{Data Preparation and Preliminary Analyses}

Missing data at the end of treatment and follow-up was higher than expected (Goodyer et al., 2017b), with 392 (84\%) participants with available data for the primary outcome of MFQ scores by the end of follow-up. As such, multiple imputation (Rubin, 1987; Sinharay, Stern, \& Russell, 2001) was used to create 20 complete data sets $(\mathrm{N}=465)$. The 
original and imputed data for repeated measures analyses were reanalysed using multilevel modelling and no difference in patterns of significance was found. The original data are reported for simplicity.

Continuous data were screened in relation to the assumptions of parametric tests (Tabachnick \& Fidell, 2007). Where assumptions were violated, confirmatory analyses were conducted by running analyses with 1000 bootstrap samples or non-parametric alternatives. All results were consistent, suggesting that the original analyses were robust to the violations of assumptions, so results based on the original (non-bootstrapped) analyses are presented for simplicity.

All medication use was recorded at baseline, and participants were required to be on a stable dose of antidepressants to take part in the trial. Of participants included in this study, $20.1 \%$ were taking antidepressants. Only one participant identified taking a sleeping medication (melatonin). Between-group differences for participants with and without medication for depression or anxiety were examined, no between-group differences were identified, and all participants were included in the following analyses. For further information on medication use and association with clinical characteristics in the present sample, see Cousins et al. (2016).

\section{Descriptive Statistics of the Nature of Sleep Problems at Baseline}

At baseline, $92 \%$ of young people described sleep difficulties that met threshold for a symptom on the K-SADS (Goodyer et al., 2017b). The most commonly reported problems at baseline were non-restorative sleep and initial insomnia, which were both reported by over half of the participants (Table 1). It was also common for adolescents to report more than one type of sleep disturbance (see Table 2), with 58\% of young people reporting 1-2 distinct sleep problems, and $35 \%$ of young people reporting between three and six different sleep problems. 
There were no differences between treatment groups for frequency of baseline sleep problems, $F(2,465)=0.53, p=0.59, \eta_{p}^{2}<0.01$.

[Insert Table 1]

\section{Pre- to Post-Treatment Change in Sleep Disturbance on Self-Report Questionnaire}

First we examined subjective ratings of sleep problems (insomnia and hypersomnia) on the adolescent MFQ at baseline, post-treatment and follow-up. To test the effects of time and treatment modality, two mixed model ANOVAs were conducted with a between-subjects independent variable of treatment arm, with three levels (BPI, STPP, and CBT), and a within subjects independent variable of time, with three levels (baseline, end of treatment and follow-up), examining mean scores for the insomnia and hypersomnia items (see Figure 1).

For insomnia there was a significant main effect of time, $V=0.43, F(2,274)=$ 103.54, $p<.001, \eta_{p}^{2}=0.43$, with ratings decreasing from baseline to follow up (Figure 1). Pairwise comparisons revealed a significant difference in insomnia between baseline and end of treatment $(p<.001)$, and baseline and follow up $(p<.001)$, but no difference between insomnia at the end of treatment and at follow-up $(p=.23)$. There was no significant main effect of treatment modality on young people's ratings of insomnia, $F(2,275)=0.27, p=.77$, $\eta_{p}{ }^{2}<0.01$. There was also no significant interaction between time and treatment arm, $V=$ $.02, F(4,550)=1.20, p=.31, \eta_{p}^{2}<0.01$.

Hypersomnia was reported infrequently on the MFQ (see Figure 1). There was no effect of time, $V=.01, F(2,275)=0.63, p=.53, \eta_{p}{ }^{2}=0.01$, treatment, $F(2,276)=0.20, p=$ $.82, \eta_{p}{ }^{2}<0.01$, or time by treatment interaction $V<.01, F(4,552)=0.12, p=.98, \eta_{p}{ }^{2}<$ 0.01 , on ratings of hypersomnia. 
[Insert Figure 1]

\section{Pre- to Post-Treatment Change in Sleep Disturbance on Diagnostic Interview}

Next, we examined symptom reports of sleep on the K-SADS clinical interview at baseline, post treatment and follow up (see Table 2). To assess if the number of sleep symptoms reduced over the course of psychological treatment for depression, we used a mixed model ANOVA with a between subjects independent variable of treatment with three levels (BPI, STPP, and CBT), and a within subjects independent variable of time with three levels (baseline, end of treatment and one year follow-up).

The number of sleep problems was significantly lower after treatment for depression $F(1.90,390.61)=106.70, p<.001$. Pairwise comparisons revealed a significant reduction in the number of sleep problems reported between baseline and end of treatment $(p<.001)$, and baseline and follow up $(p<.001)$, but not between end of treatment and follow-up $(p=.06)$. There was not a significant main effect of treatment arm, $F(2,206)=0.43, p=.65$ on the number of sleep problems reported and no significant interaction between treatment arm and time, $F(3.79,390.61)=1.13, p=.34$.

[Insert Table 2]

\section{Residual Sleep Problems}

In the original trial, 31-44\% of adolescents were still experiencing Major Depressive Disorder at the end of treatment (across the treatment arms), with 26-29\% still experiencing a diagnosis at one year follow-up (Goodyer et al., 2017b).

[Insert Table 3] 
There was a marked reduction in the frequency of sleep problems after treatment (see Tables 1 and 2); however approximately half of young people reported residual sleep difficulties at the end of treatment and at follow-up. Furthermore, of the overall sample, 33\% recovered from depression at 36 weeks but had residual problems with sleep (Table 3).

\section{Discussion}

The results of this study show that sleep problems were virtually universal amongst a large sample of depressed adolescents and that, for some young people, psychological treatments for depression appeared to improve their sleep. Although a range of different sleep disturbances were reported as part of the diagnostic interview for depression, the most common difficulties were initial insomnia (difficulty falling asleep) and non-restorative sleep (not feeling rested after sleeping). No differences in recovery of sleep disturbance were found between the three psychological therapies (CBT, STPP and BPI). These data were collected from a clinically referred group of young people, with moderate to severe Major Depressive Disorder, who were referred to and treated in routine mental health services.

These exploratory analyses indicate that according to a structured diagnostic interview for depression, approximately half of the young people did report a reduction in sleep disturbances after treatment for depression. An overall improvement was also reflected in a self-report questionnaire item measuring difficulty getting to sleep. However, this means that a substantial proportion continued to meet threshold for sleep disturbances at the end of treatment and this proportion did not change over the year of follow up. Furthermore, one third of young people recovered from their Major Depressive Disorder diagnosis, but still reported residual sleep problems after treatment and at follow up.

Given that sleep difficulties have such adverse, lasting effects on physical and psychological well-being for the individual (Shochat et al., 2014), can be disruptive for the 
family (Wake et al., 2006) and may play a causal role in the development of depression (Lovato \& Gradisar, 2014), residual sleep problems may constitute a significant on-going risk and may benefit from direct intervention. It is however also possible that for individuals that recover from depression but not from sleep disturbances, that sleep problems and depression may be distinct and not causally related.

With regards to the individuals that recover from depression and sleep disturbance, the nature of the present data and analyses makes it difficult to determine if psychological treatments improve sleep and depression independently, or if improvement in sleep is simply a bi-product of recovering from depression. Negative cognitive processes such as perseverative and ruminative thinking are pervasive present in MDD, and are hypothesised to interfere with sleep onset (Lovato \& Gradisar, 2014). Therefore, reducing perseveration and rumination may help make sleep onset more rapid. In addition, the negative information processing biases that are characteristic of MDD in adults (Joorman, Yoon, \& Zetsche, 2007) and adolescents (Orchard, Pass, \& Reynolds, 2016; Platt, Waters, Schulte-Koerne, Engelmann, \& Salemink, 2017) may lead to inflated self-report of specific sleep problems and adolescents.

There is reasonable evidence that interventions for sleep problems are effective and cost effective (Koffel et al., 2015; Trauer et al., 2015), can be delivered in different ways and made accessible through online treatment programmes (Cheng \& Dizon, 2012), and that they significantly reduce symptoms of depression (Gee et al., 2018). This evidence has mostly been collected with adults, with much less evidence that similar interventions are effective for young people, or that they are acceptable to young people or their parents. However, feasibility and pilot work in this area is growing, with recent research reporting on case studies of CBT for insomnia (CBTi) with depressed adolescents (Orchard, Pass, Chessell, Moody, Ellis \& Reynolds, in press) and the assessment of the feasibility of delivering web- 
based CBTi in child and adolescent mental health services in the UK (Cliffe, Croker, Denne, \& Stallard, 2018). Further work is needed to establish the effectiveness of psychological interventions targeting sleep in depressed young people.

The young people in this study were randomised to receive one of three different psychological interventions for depression, Cognitive Behaviour Therapy (CBT), Short Term Psychodynamic Psychotherapy (STPP), or Brief Psychosocial Intervention (BPI). CBT and STPP did not target sleep problems but BPI did include advice about sleep hygiene. Young people in all three arms reported significantly fewer problems with sleep after treatment and one year later and there was no difference between the three treatments in the reduction of sleep problems. This equivalence between treatments in reducing sleep problems is consistent with the effects of the treatments for depression severity, for which there was no significant differences in effectiveness or cost effectiveness between the three treatments (Goodyer et al., 2017b). Although BPI included some material on sleep hygiene, it is unsurprising that this treatment did not result in greater reduction of sleep disturbance given that 1) the evidence suggests sleep hygiene is not one of the active components of CBT for insomnia, 2) there is no evidence of sleep hygiene improving insomnia, and 3) in many cases sleep hygiene is used as a control treatment (e.g. Stepanski \& Wyatt, 2003; Taylor \& Roane, 2010). It is however important to acknowledge that the conclusions regarding treatment are limited by the absence of a waitlist control group and sleep problems may naturally improve over time for some individuals.

A major limitation of this study is the measurement of sleep. This limitation is somewhat attenuated by using two different measures of sleep - one based on a structured diagnostic interview by a trained assessor, the other a response to single items on a self-report questionnaire. However, because depression is associated with pervasive negative cognitive biases, perceived sleep quality may be a distortion of actual sleep quality and adolescents 
with depression may therefore significantly over-estimate their sleep problems. Similarly, reported improvements in sleep after treatment for depression may reflect a change in mood and hence perceptions of sleep, rather than any objective change in sleep quality or duration. Future work in this area should include validated questionnaire and diary measures of sleep (e.g. Insomnia Severity Index, Pittsburgh Sleep Quality Index, Consensus Sleep Diary) as well as measures that do not rely on self-report (e.g. actigraphy or polysomnography). The addition of a direct index of sleep duration, sleep variability, wakefulness, time to sleep and time awake, would help unpack the meaning of these data and untangle the subjective experience of depression and low mood, and perceived poor sleep quality.

It is also important to note that there are challenges with considering the role of sleep problems as both symptom and causal factor in depression. There is a growing body of literature suggesting that sleep problems and depression may both be caused by a separate underlying factor e.g. rumination (Lovato \& Gradisar, 2014), with a recent review considering a wide range of mechanisms which could result in associations between depression, anxiety and insomnia (Blake et al., 2018).

Relatedly, future work should also look to diagnose comorbid sleep disorders in addition to depressive disorders, as it is likely that some adolescents with MDD may also have comorbid insomnia disorder, or other sleep disorders. In the present study, residual sleep symptoms may indicate untreated sleep disorders. With some emerging evidence that disorders of insomnia and depression may have distinct characteristics (Buysse et al., 2008), it is important that these comorbidities are examined directly when evaluating treatment outcomes for depression and sleep.

This is the first study to examine recovery of sleep disturbance following psychological treatment for depression in adolescence. Despite methodological shortcomings, we present important preliminary data that has clear treatment implications as well as 
highlighting important ways in which we can improve assessment of sleep problems in the context of depression. These clinical data highlight the almost universal and adverse impact of sleep difficulties amongst depressed young people. Findings suggest that approximately half of young people treated for depression will continue to experience residual sleep problems, and a third of young people will experience residual sleep problems even in absence of depression. 


\section{Acknowledgments}

The study was funded by the National Institute for Health Research (NIHR) Health Technology Assessment (HTA) programme (project number 06/05/01). The views expressed in this publication are those of the authors and do not necessarily reflect those of the HTA programme, NIHR, National Health Service, or the Department of Health. We thank all the child and adolescent mental health service practitioners who took part in this research, Laura Villis for her administrative support throughout, and the specialist therapists who gave their professional time to rate audiotapes as experts. 


\section{References}

Alfano, C. A., Ginsburg, G. S., \& Kingery, J. N. (2007). Sleep-related problems among children and adolescents with anxiety disorders. Journal of the American Academy of Child \& Adolescent Psychiatry, 46(2), 224-232.

APA. (2000). Diagnostic and statistical manual of mental disorders (4th ed., Text Revision). Washington, DC: Author.

APA. (2013). Diagnostic and statistical manual of mental disorders (DSM-5). Arlington: American Psychiatric Publishing.

Bertocci, M. A., Dahl, R. E., Williamson, D. E., losif, A.-M., Birmaher, B., Axelson, D., \& Ryan, N. D. (2005). Subjective sleep complaints in pediatric depression: a controlled study and comparison with EEG measures of sleep and waking. Journal of the American Academy of Child \& Adolescent Psychiatry, 44(11), 1158-1166.

Blake, M. J., Blake, L. M., Schwartz, O., Raniti, M., Waloszek, J. M., Murray, G., . . McMakin, D. L. (2018). Who benefits from adolescent sleep interventions? Moderators of treatment efficacy in a randomized controlled trial of a cognitive-behavioral and mindfulness-based group sleep intervention for at-risk adolescents. Journal of Child Psychology and Psychiatry, 59(6), 637-649.

Brent, D. A., \& Poling, K. (1997). Cognitive therapy treatment manual for depressed and suicidal youth: University of Pittsburgh Health System Services for Teens At Risk.

Buysse, D. J., Angst, J., Gamma, A., Ajdacic, V., Eich, D., \& Rössler, W. (2008). Prevalence, course, and comorbidity of insomnia and depression in young adults. Sleep, 31(4), 473-480.

Carney, C. E., Segal, Z. V., Edinger, J. D., \& Krystal, A. D. (2007). A comparison of rates of residual insomnia symptoms following pharmacotherapy or cognitive-behavioral therapy for major depressive disorder. The Journal of clinical psychiatry, 68(2), 254-260.

Cheng, S. K., \& Dizon, J. (2012). Computerised cognitive behavioural therapy for insomnia: a systematic review and meta-analysis. Psychotherapy and psychosomatics, 81(4), 206-216.

Clarke, G., \& Harvey, A. G. (2012). The complex role of sleep in adolescent depression. Child and Adolescent Psychiatric Clinics, 21(2), 385-400.

Cliffe, B., Croker, A., Denne, M., \& Stallard, P. (2018). Supported Web-Based Guided Self-Help for Insomnia for Young People Attending Child and Adolescent Mental Health Services: Protocol for a Feasibility Assessment. JMIR Research Protocols, 7(12), 1-9. doi: https://doi.org/10.2196/11324

Costello, J. E., \& Angold, A. (1988). Scales to assess child and adolescent depression - checklists, screens and nets. Journal of the American Academy of Child and Adolescent Psychiatry, 27(6), 726-737.

Cousins, L., Whitaker, K. J., Widmer, B., Midgley, N., Byford, S., Dubicka, B., ... Holland, F. (2016). Clinical characteristics associated with the prescribing of SSRI medication in adolescents with major unipolar depression. European child \& adolescent psychiatry, 25(12), 1287-1295.

Dahl, R. E., \& Puig-Antich, J. (1990). Sleep disturbances in child and adolescent psychiatric disorders. Pediatrician.

de Bruin, E. J., Bögels, S. M., Oort, F. J., \& Meijer, A. M. (2015). Efficacy of cognitive behavioral therapy for insomnia in adolescents: a randomized controlled trial with internet therapy, group therapy and a waiting list condition. Sleep, 38(12), 1913-1926.

Dombrovski, A. Y., Cyranowski, J. M., Mulsant, B. H., Houck, P. R., Buysse, D. J., Andreescu, C., . . Frank, E. (2008). Which symptoms predict recurrence of depression in women treated with maintenance interpersonal psychotherapy? Depression and anxiety, 25(12), 1060-1066.

Eaton, D. K., McKnight-Eily, L. R., Lowry, R., Perry, G. S., Presley-Cantrell, L., \& Croft, J. B. (2010). Prevalence of insufficient, borderline, and optimal hours of sleep among high school students-United States, 2007. Journal of Adolescent Health, 46(4), 399-401. 
Gee, B., Orchard, F., Clarke, E., Joy, A., Clarke, T., \& Reynolds, S. (2018). The Effect of NonPharmacological Sleep Interventions on Depression Symptoms: A Meta-Analysis of Randomised Controlled Trials. Sleep medicine reviews.

Goodyer, I. M., Reynolds, S., Barrett, B., Byford, S., Dubicka, B., Hill, J., . . Roberts, C. (2017a). Cognitive-behavioural therapy and short-term psychoanalytic psychotherapy versus brief psychosocial intervention in adolescents with unipolar major depression (IMPACT): a multicentre, pragmatic, observer-blind, randomised controlled trial. Health Technology Assessment (Winchester, England), 21(12), 1.

Goodyer, I. M., Reynolds, S., Barrett, B., Byford, S., Dubicka, B., Hill, J., . . Roberts, C. (2017b). Cognitive behavioural therapy and short-term psychoanalytical psychotherapy versus a brief psychosocial intervention in adolescents with unipolar major depressive disorder (IMPACT): a multicentre, pragmatic, observer-blind, randomised controlled superiority trial. The Lancet Psychiatry, 4(2), 109-119.

Gradisar, M., Gardner, G., \& Dohnt, H. (2011). Recent worldwide sleep patterns and problems during adolescence: a review and meta-analysis of age, region, and sleep. Sleep Medicine, 12(2), $110-118$.

Gregory, A. M., \& O'Connor, T. G. (2002). Sleep problems in childhood: a longitudinal study of developmental change and association with behavioral problems. Journal of the American Academy of Child \& Adolescent Psychiatry, 41(8), 964-971.

Hiller, R. M., Johnston, A., Dohnt, H., Lovato, N., \& Gradisar, M. (2015). Assessing cognitive processes related to insomnia: a review and measurement guide for Harvey's cognitive model for the maintenance of insomnia. Sleep medicine reviews, 23, 46-53.

Hilsenroth, M. J., Blagys, M. D., Ackerman, S. J., Bonge, D. R., \& Blais, M. A. (2005). Measuring Psychodynamic-Interpersonal and Cognitive-Behavioral Techniques: Development of the Comparative Psychotherapy Process Scale. Psychotherapy: Theory, Research, Practice, Training, 42(3), 340.

IMPACT Study CBT Sub-Group (2010). COGNITIVE BEHAVIOUR THERAPY FOR DEPRESSION IN YOUNG PEOPLE MANUAL FOR THERAPISTS [PDF File]. Retrieved from http://dev.psychiatry.cam.ac.uk.

IMPACT Study CBT Sub-Group (2010). SHORT-TERM PSYCHOANALYTIC PSYCHOTHERAPY (STPP) FOR ADOLESCENTS WITH MODERATE OR SEVERE DEPRESSION: A TREATMENT MANUAL [PDF File]. Retrieved from http://dev.psychiatry.cam.ac.uk.

Kelvin, R., Dubicka, B., Byford, S., Fonagy, P., Hill, J., Reynolds, S., ... Goodyer, I. (2010). BRIEF PSYCHOSOCIAL INTERVENTION (BPI) FOR ADOLESCENTS WITH MODERATE OR SEVERE DEPRESSION: A TREATMENT MANUAL [PDF File]. Retrieved from http://dev.psychiatry.cam.ac.uk.

Johnson, E. O., Roth, T., Schultz, L., \& Breslau, N. (2006). Epidemiology of DSM-IV insomnia in adolescence: lifetime prevalence, chronicity, and an emergent gender difference. Pediatrics, 117(2), e247-e256.

Joorman, J., Yoon, K. L., \& Zetsche, U. (2007). Cognitive inhibition in depression. Applied and Preventive Psychology, 12(3), 128-139.

Kaufman, J., Birmaher, B., Brent, D., Rao, U., Flynn, C., Moreci, P., . . Ryan, N. (1997). Schedule for affective disorders and schizophrenia for school-age children-present and lifetime version (K-SADS-PL): initial reliability and validity data. Journal of the American Academy of Child \& Adolescent Psychiatry, 36(7), 980-988.

Kennard, B., Silva, S., Vitiello, B., Curry, J., Kratochvil, C., Simons, A., .. Sweeney, M. (2006). Remission and residual symptoms after short-term treatment in the Treatment of Adolescents with Depression Study (TADS). Journal of the American Academy of Child \& Adolescent Psychiatry, 45(12), 1404-1411.

Koffel, E. A., Koffel, J. B., \& Gehrman, P. R. (2015). A meta-analysis of group cognitive behavioral therapy for insomnia. Sleep medicine reviews, 19, 6-16. 
Liu, X., Buysse, D. J., Gentzler, A. L., Kiss, E., Mayer, L., Kapornai, K., . . Kovacs, M. (2007). Insomnia and hypersomnia associated with depressive phenomenology and comorbidity in childhood depression. Sleep, 30(1), 83-90.

Lofthouse, N., Gilchrist, R., \& Splaingard, M. (2009). Mood-related sleep problems in children and adolescents. Child and Adolescent Psychiatric Clinics, 18(4), 893-916.

Lovato, N., \& Gradisar, M. (2014). A meta-analysis and model of the relationship between sleep and depression in adolescents: recommendations for future research and clinical practice. Sleep medicine reviews, 18(6), 521-529.

Martell, C. R., Addis, M. E., \& Jacobson, N. S. (2001). Depression in context: Strategies for guided action: WW Norton \& Co.

Midgley, N., Reynolds, S., Kelvin, R., Loades, M., Calderon, A., Martin, P., \& O'Keeffe, S. (2018). Therapists' techniques in the treatment of adolescent depression. Journal of Psychotherapy Integration.

Nierenberg, A., Husain, M., Trivedi, M., Fava, M., Warden, D., Wisniewski, S., .. Rush, A. (2010). Residual symptoms after remission of major depressive disorder with citalopram and risk of relapse: a STAR* D report. Psychological medicine, 40(1), 41-50.

Ohayon, M. M., Roberts, R. E., Zulley, J., Smirne, S., \& Priest, R. G. (2000). Prevalence and patterns of problematic sleep among older adolescents. Journal of the American Academy of Child \& Adolescent Psychiatry, 39(12), 1549-1556.

Orchard, F., Pass, L., Chessell, C., Moody, A., Ellis, J. \& Reynolds, S. (in press). Adapting Brief CBT-I for Depressed Adolescents: A Case Illustration of the Sleeping Better Programme. Cognitive and Behavioral Practice.

Orchard, F., Pass, L., Marshall, T., \& Reynolds, S. (2017). Clinical characteristics of adolescents referred for treatment of depressive disorders. Child and Adolescent Mental Health, 22(2), 61-68.

Orchard, F., Pass, L., \& Reynolds, S. (2016). 'It Was All My Fault'; Negative Interpretation Bias in Depressed Adolescents. Journal of Abnormal Child Psychology, 44(5), 991-998.

Owens, J. (2014). Insufficient sleep in adolescents and young adults: an update on causes and consequences. Pediatrics, 134(3), e921-e932.

Peterman, J. S., Carper, M. M., Elkins, R. M., Comer, J. S., Pincus, D. B., \& Kendall, P. C. (2016). The effects of cognitive-behavioral therapy for youth anxiety on sleep problems. Journal of Anxiety Disorders, 37, 78-88.

Platt, B., Waters, A. M., Schulte-Koerne, G., Engelmann, L., \& Salemink, E. (2017). A review of cognitive biases in youth depression: attention, interpretation and memory. Cognition and emotion, 31(3), 462-483.

Ritschel, L. A., Ramirez, C. L., Jones, M., \& Craighead, W. E. (2011). Behavioral activation for depressed teens: A pilot study. Cognitive and Behavioral Practice, 18(2), 281-299.

Roane, B. M., \& Taylor, D. J. (2008). Adolescent insomnia as a risk factor for early adult depression and substance abuse. Sleep, 31(10), 1351-1356.

Roberts, R. E., \& Duong, H. T. (2013). Depression and insomnia among adolescents: a prospective perspective. Journal of Affective Disorders, 148(1), 66-71.

Rubin, D. B. (1987). Multiple imputation for survey nonresponse: New York: Wiley.

Shochat, T., Cohen-Zion, M., \& Tzischinsky, O. (2014). Functional consequences of inadequate sleep in adolescents: a systematic review. Sleep medicine reviews, 18(1), 75-87.

Sinharay, S., Stern, H. S., \& Russell, D. (2001). The use of multiple imputation for the analysis of missing data. Psychological methods, 6(4), 317.

Sivertsen, B., Harvey, A. G., Pallesen, S., \& Hysing, M. (2017). Trajectories of sleep problems from childhood to adolescence: a population-based longitudinal study from Norway. Journal of sleep research, 26(1), 55-63.

Staner, L. (2010). Comorbidity of insomnia and depression. Sleep medicine reviews, 14(1), 35-46. 
Stepanski, E. J., \& Wyatt, J. K. (2003). Use of sleep hygiene in the treatment of insomnia. Sleep medicine reviews, $7(3), 215-225$.

Tabachnick, B. G., \& Fidell, L. S. (2007). Experimental designs using ANOVA: Thomson/Brooks/Cole.

Tang, T. Z., DeRubeis, R. J., Beberman, R., \& Pham, T. (2005). Cognitive changes, critical sessions, and sudden gains in cognitive-behavioral therapy for depression. Journal of consulting and clinical psychology, 73(1), 168.

Taylor, D. J., \& Roane, B. M. (2010). Treatment of insomnia in adults and children: a practice-friendly review of research. Journal of clinical psychology, 66(11), 1137-1147.

Trauer, J. M., Qian, M. Y., Doyle, J. S., Rajaratnam, S. M., \& Cunnington, D. (2015). Cognitive behavioral therapy for chronic insomnia: a systematic review and meta-analysis. Annals of internal medicine, 163(3), 191-204.

Urrila, A. S., Karlsson, L., Kiviruusu, O., Pelkonen, M., Strandholm, T., \& Marttunen, M. (2012). Sleep complaints among adolescent outpatients with major depressive disorder. Sleep Medicine, 13(7), 816-823.

van Straten, A., van der Zweerde, T., Kleiboer, A., Cuijpers, P., Morin, C. M., \& Lancee, J. (2017). Cognitive and behavioral therapies in the treatment of insomnia: A meta-analysis. Sleep medicine reviews.

Wake, M., Morton-Allen, E., Poulakis, Z., Hiscock, H., Gallagher, S., \& Oberklaid, F. (2006). Prevalence, stability, and outcomes of cry-fuss and sleep problems in the first 2 years of life: prospective community-based study. Pediatrics, 117(3), 836-842.

WHO. (1992). The ICD-10 classification of mental and behavioural disorders: clinical descriptions and diagnostic guidelines (Vol. 1): World Health Organization.

Wittmann, M., Dinich, J., Merrow, M., \& Roenneberg, T. (2006). Social jetlag: misalignment of biological and social time. Chronobiology international, 23(1-2), 497-509.

Wu, J. Q., Appleman, E. R., Salazar, R. D., \& Ong, J. C. (2015). Cognitive behavioral therapy for insomnia comorbid with psychiatric and medical conditions: a meta-analysis. JAMA internal medicine, 175(9), 1461-1472. 
Table 1. Frequency of K-SADS sleep disturbances at baseline, end of treatment and followup

\begin{tabular}{lccc}
\hline \% & Baseline & End of Treatment (36 weeks) & Follow-up (86 weeks) \\
& $(\boldsymbol{n}=\mathbf{4 6 5})$ & $(\boldsymbol{n}=\mathbf{2 7 0})$ & $(\boldsymbol{n}=\mathbf{2 8 2})$ \\
\hline Initial Insomnia & 53.3 & 24.2 & 20.9 \\
Middle Insomnia & 32.5 & 11.9 & 11.5 \\
Terminal Insomnia & 17.6 & 14.4 & 10.2 \\
Circadian Reversal & 23.9 & 12.8 & 13.3 \\
Non-Restorative & 68.0 & 27.0 & 18.0 \\
Hypersomnia & 15.1 & 9.7 & 11.9 \\
\hline Any sleep problem & 91.8 & 55.6 & 47.2 \\
\hline
\end{tabular}


Table 2. Frequency of K-SADS sleep disturbances at baseline, end of treatment and follow$\underline{\operatorname{up}(\%)}$

\begin{tabular}{|c|c|c|c|}
\hline No. Sleep & Baseline & End of Treatment (36 weeks) & Follow-up (86 weeks) \\
\hline Disturbances & $(n=465)$ & $(n=270)$ & $(n=282)$ \\
\hline 0 & 8 & 44 & 53 \\
\hline $1-2$ & 58 & 44 & 37 \\
\hline $3-4$ & 32 & 11 & 10 \\
\hline $5-6$ & 3 & 0 & 0 \\
\hline
\end{tabular}


Table 3. Frequencies of participants with and without a diagnosis of depression and residual $\underline{\text { sleep symptom at post-treatment }(n=270)}$

\begin{tabular}{ll|ll} 
& & \multicolumn{2}{|l}{ Major Depressive Disorder } \\
& & Yes & No \\
\hline Sleep Symptom & Yes & $62(23.0 \%)$ & $88(32.6 \%)$ \\
& No & $3(1.1 \%)$ & $117(43.3 \%)$
\end{tabular}


Figure 1. Mean MFQ item ratings of insomnia and hypersomnia items at baseline $(n=462)$, end of treatment $(n=317)$ and 1-year follow-up $(n=349)$

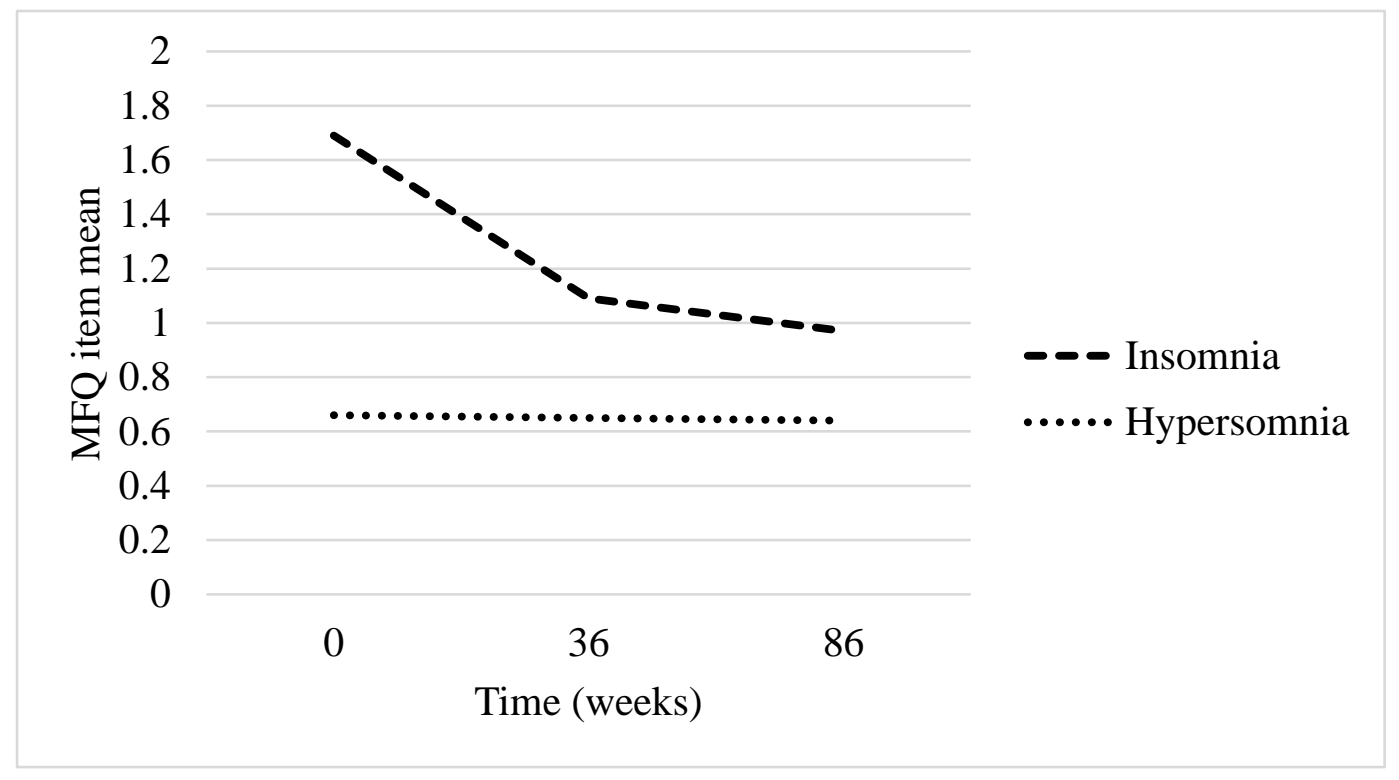

